\title{
Optimal Pricing for Spectrum Allocation in Cognitive Radio Networks
}

\author{
Fan $\mathrm{Gu}^{1}$, Xianwei $\mathrm{Li}^{2, \mathrm{a}}$, Liang Zhao ${ }^{3}$, Haiyang Zhang ${ }^{4}$, and Xiaoying Yang ${ }^{5}$ \\ ${ }^{1}$ School of Continuing Education, Anhui Institute of International Business, Hefei, China \\ ${ }^{2}$ School of Information Engineering, Suzhou University, Suzhou, China \\ ${ }^{3}$ School of School of Computer Science, Shenyang Aerospace University, Shenyang, China \\ ${ }^{4}$ School of Environment and Geomatics Engineering, Suzhou University, Suzhou, China \\ ${ }^{5}$ School of Information Engineering, Suzhou University, Suzhou, China
}

\begin{abstract}
In this paper, we investigate the problem of spectrum allocation in Cognitive Radio Networks (CRNs) where a primary user (PU) leases its idle spectrums to a number of secondary users (SUs) who may have different willing to pay for these spectrums. For the PU, its main objective is to gain profit from selling the idle spectrums, and from the perspective of the Sus, they aim to maximize their utility from using spectrums. We model the interaction between the PU and SUs as a two-stage Stackelberg game, where the PU sets the prices for its spectrum in the first stage, and SUs make their spectrum demands decisions in the second stage. We use the backward induction method to solve this game. The numerical results show that the proposed solution method can increase revenue of the PU compared with the uniform pricing scheme.
\end{abstract}

\section{Introduction}

Radio spectrum is recognized as one of the most scare and precious resources in the wireless networks, which is conventionally controlled by government via static license-based allocations. However, according to some recent works, many spectrum bands are not fully utilized even in densely populated urban areas, such Chicago [1][3]. Besides, users' demands for wireless data service have increased rapidly in the past few years. According to a recent report from by Cisco, the monthly global mobile data traffic will reach 49 exabytes by 2021 [4], as shown in Figure 1. The paradox between the rapidly growing demand for wireless data services and low utilization of spectrum allocation suggests that there are some shortcomings for current static spectrum allocation policy.

Cognitive Radio (CR), also known as dynamic spectrum access (DSA), has been proposed as a novel approach to improve the efficiency utilization of spectrum. In Cognitive Radio Networks (CRNs), unlicensed secondary users (SUs) can lease the unused spectrum bands from licensed primary users (PUs) and then provide network services to mobile users.

Nowadays, mobile virtual network operators (MVNOs) have received significant success worldwide, and have attracted a lot of research interest in the engineering and academic communities. One characteristic of MVNOs is that they do not own the physical infrastructure and they lease spectrum from Pus to provide network services to mobile users. For example,

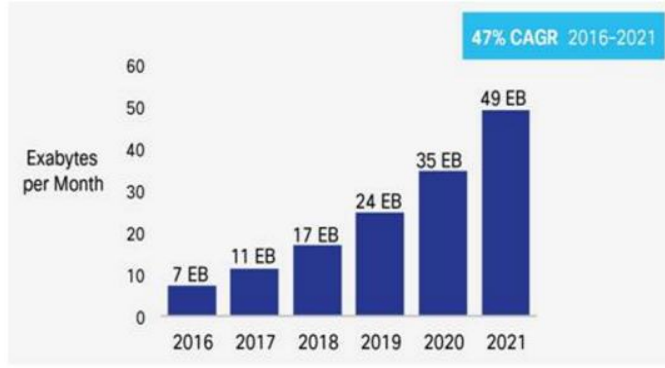

Source: Cisco VNI Mobile, 2017

Figure 1. The increase of mobile data traffic [4].

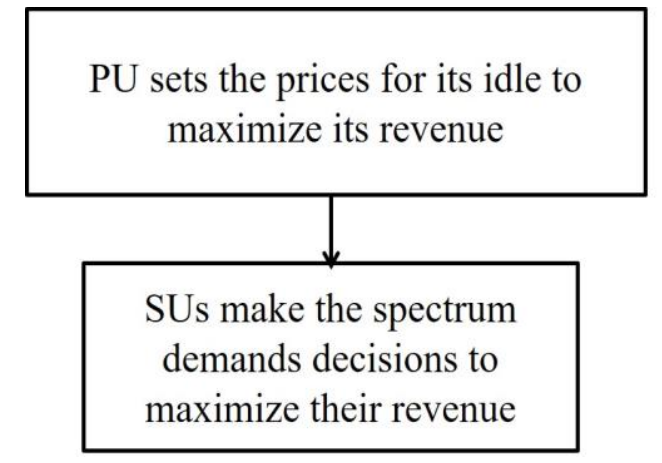

Figure 2. Two-level structure between PU and SUs.

IIJmio and LINE MOBILE are two MVNOs in Japan, and Karma is a MVNO in USA.

\footnotetext{
a Corresponding author: lixianwei163@163.com
} 


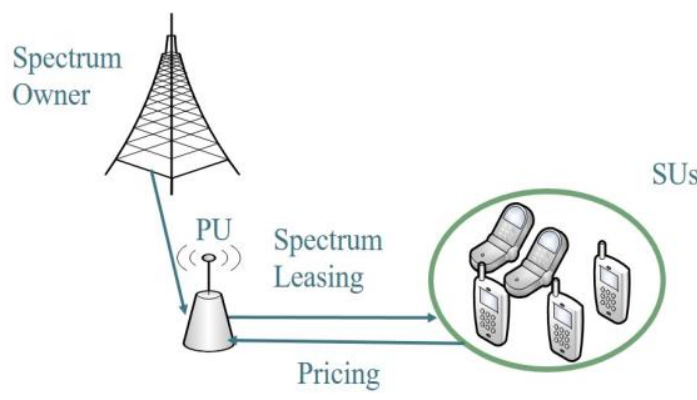

Figure 3. System Model.

Although a significant amount of attention has been paid to spectrum allocation in CRNs, most of the existing works focus on the technical aspects of spectrum sharing (e.g., designing power control method). In this paper we study from the economic aspect. Additionally, different from previous works that simply analyse homogeneous SUs, namely all the SUs have the same willing to pay for using the spectrum service, we consider heterogeneous types of SUs. For example, some SUs who are watching videos may have higher valuations of the spectrum while some other SUs who are just phoning have lower valuations on the spectrum [5].

In this paper, we investigate spectrum allocation in CRNs where SUs, such as IIJmio in Japan, lease the unused spectrums from the PU and provide network services to mobile users. The objective of the PU is to make revenue from selling the idle spectrums, and the SUs aim to maximize the utility of them. We model the interaction between the two operators and SUs as a Stakelberg (leader-follower) game, where the two operators first set service access prices to maximize their profits and then each SU will decide which operator to select based on prices and spectrum quality, as illustrated in Fig.2. We solve the game by using the backward induction method.

\section{Related Works}

Our work is related to the works that focus on spectrum allocation in CRNs.

Game theory is an effective tool for spectrum allocation in wireless networks. Some works focus on the duopoly communications market. For example, Ren et al. studied the problem of long-term entry and spectrum sharing scheme decision from the perspective of an entrant network service provider [9]. Due to users have different preferences for different time slots, Zhang et al. studied time-dependent price competition in a duopoly wireless networks market [10].

Spectrum trading and sharing in CRNs have been extensively studied with game theory. Some works related works analysed the interaction between the primary and secondary operators. For example, the authors in [7] jointly addressed the problem of pricing and network selection in CRNs, where the primary operator who can provide higher guaranteed service and the secondary operator who provides cheaper besteffort secondary network service compete to serve a common pool of users. The problem under consideration is formulated as a a Stackelberg game, where the two operators first set the prices of network services to maximize their revenues. Then, users decide which operator to select. Duan et al. studied duopoly price competition in CRNs, where two SUs compete to lease spectrum from a spectrum holder [11]. However, users' heterogeneous types are not considered and the interference factor is not fully analysed in these previous works. Different from the aforementioned works, we take both users' heterogeneous types and channel inteference into consideration. In particular, we propose a pricing scheme that charges different types of SUs with different prices. Compared with the uniform pricing scheme that assumed all SU have homogeneous valuations for the spectrum, the proposed pricing scheme can increase the revenue of the PU significantly.

\section{System Model}

In this section, we introduce the system model. As the radio spectrum allocated to PUs remains largely unused even in densely populated urban areas [1], the unlicensed SUs can lease the unused spectrum and then provide network services to mobile users. We consider a CRN with one PU, and the number of $N$ SUs. These SUs lease spectrum from the PU, as illustrated in Fig.3.

\subsection{SUs' Model}

For SU $i, i \in\{1,2, \ldots, N\}$, its type is denoted by $\theta_{i}$ which reflects SUs' heterogeneous willing to pay for the spectrum of PU. Without loss of generality, we assume that $\theta_{1}<\theta_{2} \ldots<\theta_{N}$. The system model that we use is mainly inspired by [8] and [11] but with different pricing schemes.

For the SU $i$, if it obtains spectrum from $w_{i}$ from the $\mathrm{PU}$, it can achieve the data rate

$$
R_{\mathrm{i}}=w_{i} \log _{2}\left(1+r_{i}\right)
$$

where $r_{i}$ is the SINR that SU $i$ received and is denoted as

$$
r_{\mathrm{i}}=\frac{p_{i} h_{i, i}}{\sum_{j=1, j \neq i}^{N} p_{j} h_{j, i}+\sigma_{i}^{2}}
$$

where $p_{i}$ is the $\mathrm{SU} i$ 's transmission power, $h_{i, j}$ is the channel gain from $\mathrm{SU} i$ to $\mathrm{SU} j$, and $\sigma^{2}$ is the noise variance.

For the SU $i$, if its obtains spectrum from $w_{i}$ from the PU, it will get the utility

$$
u_{\mathrm{i}}=\theta_{i} w_{i} \log _{2}\left(1+r_{i}\right)-\pi_{i} w_{i}
$$


where, $\pi_{i}$ is the price charged with SU $i$ per unit spectrum. Note that different from many of the existing works that charge all the users with the same price, we set different types of SUs with different prices, which if more practical.

\subsection{PU's Model}

Assume the a PU in a CRN has temporally idle spectrum $B$. From the perspective of the PU, its objective is to sell the unused spectrum to SUs to make profits. For the PU, the profit of selling $w_{i}$ is denoted as

$$
R_{i}=\left(\pi_{i}-c\right) w_{i}
$$

where $c$ is the cost of purchasing unit spectrum from the spectrum holder.

Accordingly, the total profit of the PU from selling spectrum to the SUs is denoted as

$$
R=\sum_{i=1}^{N}\left(\pi_{i}-c\right) w_{i}
$$

For convenience of analysis, the notations used throughout this paper are summarized in Table 1.

\section{Two-Stage Stackelberg Game}

In this section, we analyse the interaction between the PU and SUs, which is modelled as two-stage Stackelberg game. In this game, the PU sets the prices for the spectrum in the first stage, and the SUs make spectrum demand decisions in the second stage. We consider complete information case which means that the PU knows the type of each SU. We try to solve this game by employing the backward induction method [14], [15]. We first analyse the utility maximization of SUs in Stage II based on the price of network service. Then, we investigate how the secondary operator sets network service prices in Stage I.

Table 1. Notations Summary.

\begin{tabular}{|c|c|}
\hline Notation & Description \\
\hline$i$ & subscript of a SU \\
\hline$\pi_{i}$ & $\begin{array}{c}\text { the price per spectrum charged } \\
\text { with SU } i\end{array}$ \\
\hline$R_{i}$ & $\begin{array}{c}\text { the profit of PU for selling } \\
\text { spectrum } w_{i} \text { to user } i\end{array}$ \\
\hline$R_{i}^{*}$ & optimal profit of the PU \\
\hline$B$ & $\begin{array}{c}\text { the total idle spectrum of the PU } \\
\text { the type of SU } i \text {, which reflects this } \\
\text { user's willing to pay for spectrum }\end{array}$ \\
\hline$\theta_{i}$ & $\begin{array}{c}\text { the purchased spectrum of SUs' } \\
\text { from PU }\end{array}$ \\
\hline$W_{i}$
\end{tabular}

\begin{tabular}{|c|c|}
\hline$c$ & $\begin{array}{c}\text { the cost of PU for leasing per unit } \\
\text { spectrum from spectrum holder }\end{array}$ \\
\hline$p_{i}$ & the SU $i$ 's transmission power \\
\hline$h_{i, j}$ & $\begin{array}{c}\text { the channel gain from SU } i \text { to } \\
\text { SU } j\end{array}$ \\
\hline$\sigma^{2}$ & the noise variance \\
\hline$r_{i}$ & the SINR that SU $i$ received \\
\hline$u_{i}$ & $\begin{array}{c}\text { the utility that type } \theta_{i} \text { SU gets from } \\
\text { buying spectrum from PU }\end{array}$ \\
\hline
\end{tabular}

\subsection{SU's Utility Maximization Problem in Stage II}

For the SU $\theta_{i}$, it will subscribe to network services if and only if

$$
u_{i}=\theta_{i} w_{i} \log _{2}\left(1+r_{i}\right)-\pi_{i} w_{i} \geq 0
$$

From which, we know that the price of the spectrum set for SU $i$ should meet the following requirement:

$$
\pi_{i} \leq \theta_{i} \log _{2}\left(1+r_{i}\right)
$$

It is obvious that the optimal price is

$$
\pi_{i}^{*}=\theta_{i} \log _{2}\left(1+r_{i}\right)
$$

\subsection{PU's Profit Maximization Problem in Stage I}

From the perspective of the PU, its objective is to maximize profit, which is the difference between its revenue and cost. The optimal profit for selling the number of $w_{i}$ is

$$
R_{\mathrm{i}}^{*}=\left(\pi_{i}^{*}-c\right) w_{i}=\left(\theta_{i}^{*} \log _{2}\left(1+r_{i}\right)-c\right) w_{i}
$$

Therefore, the total optimal profit that PU gets from selling idle spectrum to SUs is denoted as

$$
R^{*}=\sum_{i=1}^{N}\left(\theta_{i} \log _{2}\left(1+r_{i}\right)-c\right) w_{i}
$$

\section{Numerical Results}

In this section, we present numerical results to validate the performance of our proposed pricing scheme. The parameters are set as follows unless otherwise specified: $N=5, \theta_{i}=i, w_{i}=1$ and $r_{i}=i$.

We first compare the proposed pricing scheme considering heterogeneous SU types with the case when all the SUs have homogeneous type, that is, all SUs have the same willing to pay for spectrum. From Fig.4 we observe that the PU can get more profits if SUs' heterogeneous types are taken into account. This figure also suggests that the increase in cost of spectrum will lead to profit loss for the PU. 


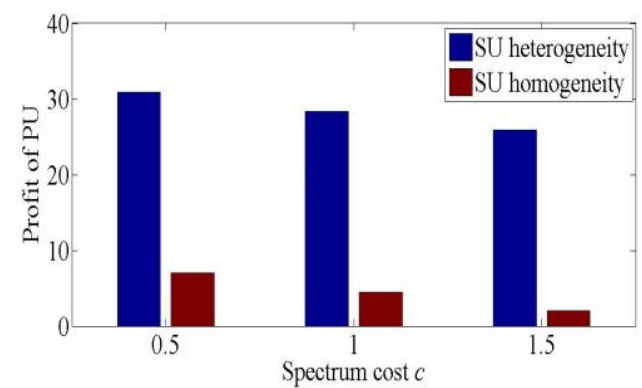

Figure 4. Compared with the homogeneous SU

We next analyse the utility of SUs. From Figure 5, we can observe that SU 5 can obtain more utility than SU 2 due to the reason that SU 5 has a higher value, which means that this user is more willing to pay to the spectrum of PU. This figure indicates that PU can easily sell spectrum to SUs who are more willing to pay for using spectrum. From Figures 4 and 5, we can obviously observe that $\mathrm{PU}$ can make use of the heterogeneity to get more profits from selling idle spectrums.

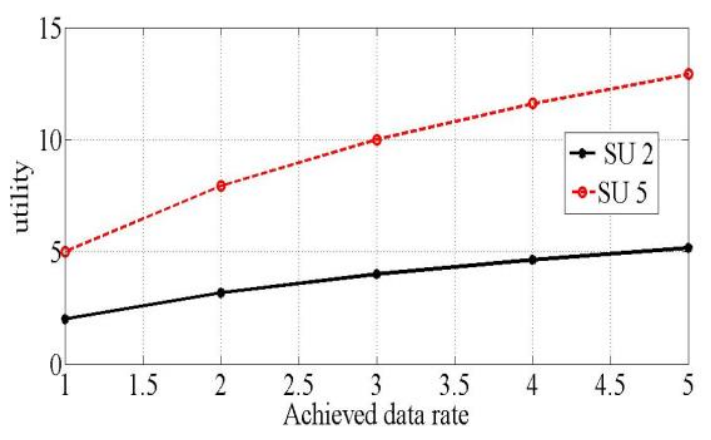

Figure 5. Compare the utility of SU 2 and 5.

\section{Conclusions}

In this paper, we studied price differentiation in a CRN, where the idle spectrums of PU are leased to SUs, and SUs provide network service mobile users with the objective of maximizing their utility. Our numerical results show compared with the uniform pricing scheme, the proposed price differentiation scheme can improve the profit of the PU greatly.

\section{Acknowledgement}

This work is partly supported by Natural science research project of University of Anhui Province (KJ2016A125), the National Science Foundation for Young Scientists of China (61701322), the Key Projects of Liaoning Natural Science Foundation (20170540700), the Liaoning Provincial Department of Education Science Foundation (L201630), the project of College Students Construction Plan of Anhui Province (s2015ckjh150), the Major Project of Natural Science of Education Department of Anhui Province (KJ2014ZD31), Key Scientific Research Projects of Suzhou University (2016yzd10, 2015ykf13), Key projects of the youth talent support program of Suzhou University (2016YQNRC004), and Suzhou Regional Collaborative Innovation Center (2016szxt05, 2016szxt06).

\section{References}

1. L. Duan, J. Huang, and B. Shou, Duopoly competition in dynamic spectrum leasing and pricing, IEEE T. Mobile Comput., vol.11, no.11, pp.1706-1719, Nov.2012.

2. L. Duan, J. Huang, and B. Shou, Investment and pricing with spectrum uncertainty: A cognitive operator's perspective, IEEE T. Mobile Comput., vol.10, no.11, pp.1590-1604, Nov.2011.

3. M. S.Khan, M. Usman, and V.V.Hiep et al., Efficient selection of users pair in cognitive radio network to maximize throughput using simultaneous transmit-sense approach, IEICE Trans. Commmu., vol.E100-B, no.2, pp.380-389, Feb.

4. Ciscovisualnetworkingindex:Forecastandmethodol ogy, 2016-2021. https://www.cisco.com/c/en/us/solutions/collateral / service-provider/visual-networking-indexvni/mobile-white-paper-c11-520862.html.

[ accessed on Auguset 10, 2017].

5. X. Cao, Y. Chen, and K.J.R. Liu, Cognitive radio networks with heterogeneous users: How to procure and price the spectrum?, IEEE T. Commun., vol.14, no.3, pp.1676-1688, March 2015.

6. D. Niyato and E. Hossain, A game theoretic analysis of service competition and pricing in heterogeneous wireless access networks, IEEE T. Commun., vol.7, no.12, pp.5150-5155, Dec.2008.

7. J. Elias, F. Martignon, and L. Chen et al., Joint operator pricing and network selection game in cognitive radio networks: Equilibrium, system dynamics and price of anarchy, IEEE T. Veh Technol., vol.62, no.9, pp.4576-4589, Nov.2013.

8. F. Li, Z. Sheng, and J. Hua et al, Preference-based spectrum pricing in dynamic spectrum access networks, IEEE T. Serv. Comput., in press.

9. S.Ren, K.Park, andM.Schaar, Entry and spectrum sharings cheme selection in femtocell communications markets, IEEE/ACM Trans. Netw., vol.21, no.1, pp.218-232, Feb. 2013.

10. C. Zhang, B. Gu, and K. Yamori et al., Duopoly competition in time-dependent pricing for improving revenue of network service providers, 
IEICE Trans. Commmu., vol.E96-B, no.12, pp.2964-2975, Dec. 2015.

11. L. Duan, J. Huang, and B. Shou, "Duopoly competition in dynamic spectrum leasing and pricing," IEEE T. Mobile Comput., vol.11, no.11, pp.1706-1719, Nov.2012.

12. N.H. Tran, C.S. Hong, and Z. Han et al., Optimal pricing effect on equilibrium behaviors of delaysensitive users in cognitive radio networks, IEEE Trans. J. Sel. Areas Commun., vol.31, no.11, pp.2566-2579, Nov. 2013.

13. N.H. Tran, L.B. Le, S. Ren, Z. Han, and C.S. Hong, Joint pricing and load balancing for cognitive spectrum access: Non-cooperation versus cooperation, IEEE Trans. J. Sel. Areas Commun., vol.33, no.5, pp.972-985, May 2015.

14. D. Fudenberg and J. Tirole, Game theory, MIT Press, Cambridge, MA, USA, 1991.

15. Z. Han, D. Niyato, and W. Saad et al., Game theory in wireless and communication networks: Theory, models, and applications, Cambridge University Press, Cambridge, UK, 2011.

16. S. Zhao, Q. Zhu, and G. Zhu et al., Competitions and dynamics of mvnos in spectrum sharing: An evolutionary game approach, IEICE Trans. Commmu., vol.E96-B, no.1, pp.69-72, Jan.2013.

17. X.J. Tan, L. Li, and W. Guo, A game-theoretic approach for opportunistic spectrum sharing in cognitive radio networks with incomplete information, IEICE Trans. Commmu., vol.95, no.4, pp.1117-1124, April 2012.

18. L. Gao, X. Wang, Y. Xu, and Q. Zhang, Spectrum trading in cognitive radio networks: A contracttheoretic modeling approach, IEEE J. Sel. Areas Commun., vol. 29, no. 4, pp. 843-855, April 2011. 